\title{
Density Fluctuations in Fluids Having an Internal Degree of Freedom*
}

\author{
Raymond D. Mountain
}

\author{
Institute for Basic Standards, National Bureau of Standards, Washington, D.C. 20234
}

(August 18, 1967)

\begin{abstract}
The frequency spectrum of density fluctuations is calculated for a fluid whose molecules possess an internal degree of freedom which is weakly coupled to the translational degree of freedom of the fluid. Irreversible thermodynamics is used to obtain an equation of motion for the internal degree of freedom. This equation plus the linearized hydrodynamic equations are solved for the frequency spectrum of density fluctuations. The results are compared with a similar calculation involving a frequency dependent volume viscosity. The results are identical for structural relaxation but there is a difference for thermal relaxation. The origin of the difference is discussed and the magnitude of the difference is examined for $\mathrm{CCl}_{4}$ and for $\mathrm{CS}_{2}$.
\end{abstract}

Key Words: Brillouin scattering, density fluctuations in liquids, Rayleigh scattering, spectral distribution of scattered light, structural relaxation, thermal relaxation, volume viscosity.

\section{Introduction}

The spectrum of light scattered by density fluctuations in a fluid is proportional to frequency spectrum of density fluctuations [1]. ${ }^{1}$ The development of the gas laser has made it possible to measure the spectra of long wavelength density fluctuations and thereby study fluids in the frequency region of a few gigacycles down to essentially zero cycles/sec.

For dense fluids this frequency region corresponds to slowly varying processes whose time dependence may be reasonably expected to be described by the equations of irreversible thermodynainics. When considering density fluctuations the appropriate equations are the linearized equations of hydrodynamics plus two additional relations among the variables so that a solution to the initial value (or boundary value) problem is possible.

In this paper we shall consider density fluctuations in a fluid whose molecules possess an internal degree of freedom which relaxes toward local equilibrium with the density and/or the temperature [2,3]. This is carried out in section 2. In section 3 this calculation is compared with an earlier calculation [1] where the internal degree of freedom was assumed to result in a frequency dependent volume viscosity. The introduction of a frequency dependent volume viscosity is based on statistical mechanical arguments $[4,5]$.

\footnotetext{
*This work was sponsored by the Advanced Research Projects Agency of the Department of Defense.

${ }^{1}$ Figures in brackets indicate the literature references at the end of this paper.
}

The comparison is made in two limiting cases. In the first case it is assumed that the internal variable depends only on the local temperature (thermal relaxation). It is found that the two calculations yield similar, but not identical, results. The origin and magnitude of the difference are examined. Carbon tetrachloride and carbon disulfide are used to illustrate the discussion. In the second case it is assumed that the internal variable depends only on the local density (structural relaxation). The two calculations yield identical results.

No comparison is made when both thermal relaxation and structural relaxation are present.

\section{Calculation}

The thermodynamic statement relating the parameters for our system is [6]

$$
d U=T d S+\frac{p}{\rho_{0}^{2}} d \rho+A_{\xi} d \xi
$$

where $U$ is the energy, $S$ is the entropy, $T$ is the temperature, $\rho$ is the density, $\rho_{0}$ is the equilibrium value of the density, $p$ is the pressure, $\xi$ represents an internal degree of freedom and $A_{\xi}$ is the partial derivative of the Helmholtz free energy with respect to $\xi$,

$$
A_{\xi}=(\partial A / \partial \xi)_{\rho, T} .
$$

We shall further require that $A_{\xi}=0$ when the internal degree of freedom is in local equilibrium with the 
density and the temperature (whether or not the density and temperature have reached equilibrium values). This may be expressed as

$$
A_{\xi}(\rho, T, \bar{\xi})=0
$$

where

$$
\bar{\xi}=\xi(\rho, T)
$$

is the local equilibrium value of $\xi$.

A kinetic equation for $\xi$ is obtained by applying the methods of irreversible thermodynamics to the system with the result

$$
\partial \xi / \partial t=-L A_{\xi}
$$

where the kinetic coefficient $L$ is $>0$. As we are concerned with small deviations from equilibrium, $A_{\xi}$ is expanded to obtain

$$
\frac{\partial \xi}{\partial t}=-L\left[A_{\xi \rho}\left(\rho-\rho_{0}\right)+A_{\xi, T}\left(T-T_{0}\right)+A_{\xi \xi}\left(\xi-\xi_{0}\right)\right]
$$

where $A_{\xi \rho}=\left(\partial A_{\xi} / \partial \rho\right)_{T, \xi}$, etc. If we expand eq (3) and substitute this in eq (6) we obtain

$$
\partial \xi / \partial t=-L A_{\xi \xi}(\xi-\bar{\xi}) .
$$

The equations of motion for the system are the linearized hydrodynamic equations:

$$
\begin{gathered}
\partial \rho / \partial t+\rho_{0} \psi=0 \\
m \rho_{0} \partial \psi / \partial t=-\nabla^{2} p+\left(\frac{4}{3} \eta_{s}+\eta_{v}\right) \nabla^{2} \psi
\end{gathered}
$$

and

$$
\rho_{0} T_{0} \partial S / \partial t=\lambda \nabla^{2} T \text {. }
$$

Here $\psi=\operatorname{div} \mathbf{v}, \eta_{s}$ is the shear viscosity, $\eta_{v}$ is the volume viscosity and $\lambda$ is the thermal conductivity and $m$ is the mass of a molecule. We relate the pressure and entropy to the density, temperature and $\xi$ by the thermodynamic relations

$$
d p=\left(\frac{\partial p}{\partial \rho}\right)_{T, \xi} d \rho+\left(\frac{\partial p}{\partial T}\right)_{\rho, \xi} d T+\left(\frac{\partial p}{\partial \xi}\right)_{\rho, T} d \xi
$$

and

$$
d S=\left(\frac{\partial S}{\partial \rho}\right) d \rho+\left(\frac{\partial S}{\partial T}\right)_{\rho, \xi} d T+\left(\frac{\partial S}{\partial \xi}\right)_{T, \rho} d \xi
$$

The internal variable $\xi$ is eliminated by combining eqs (6), (11), and (12). For the pressure we find

$$
d p+\tau \frac{\partial d p}{\partial t}=\left(\frac{\partial p}{\partial \rho}\right)_{T, \xi}\left[d \rho+\tau \frac{\partial d \rho}{\partial t}\right]
$$

$$
\begin{aligned}
& +\left(\frac{\partial p}{\partial T}\right)_{\rho, \xi}\left[d T+\tau \frac{\partial d T}{\partial t}\right] \\
& +\left(\frac{\partial p}{\partial \xi}\right)_{\rho, T}\left[-\frac{A_{\xi \rho}}{A_{\xi \xi}} d \rho-\frac{A_{\xi T}}{A_{\xi \xi}} d T\right]
\end{aligned}
$$

where

$$
\tau=\left(L A_{\xi \xi}\right)^{-1} .
$$

A similar equation is obtained for the entropy.

$$
\begin{aligned}
d S & +\tau \frac{\partial d S}{\partial t}=\left(\frac{\partial S}{\partial \rho}\right)_{T, \xi}\left[d \rho+\tau \frac{\partial d}{\partial t}\right] \\
& +\left(\frac{\partial S}{\partial T}\right)_{\rho, \xi}\left[d T+\tau \frac{T}{\partial t}\right] \\
& +\left(\frac{\partial S}{\partial \xi}\right)_{\rho, T}\left[-\frac{A_{\xi \rho}}{A_{\xi \xi}} d \rho-\frac{A_{\xi T}}{A_{\xi \xi}} d T\right] .
\end{aligned}
$$

Equations (8), (9), (10), (13), and (14) constitute a complete set of equations. The next step is to obtain the Fourier-Laplace (space-time) transform of this set of equations. The result is

$$
z \rho_{k}(z)+\rho_{0} \psi_{k}(z)=\rho_{k},
$$

$$
\begin{aligned}
\left(z+b_{0} k^{2}\right) \psi_{k}(z) & -\left[\frac{C_{0}^{2} k^{2}}{\rho_{0}}+\frac{\Delta k^{2}}{\rho_{0}} \cdot \frac{z \tau}{1+z \tau}\right] \rho_{k}(z) \\
& -\left[\frac{C_{0}^{2} k^{2} \beta_{T}}{\gamma}+\frac{\rho_{0}}{m} A_{\xi \xi} \xi_{\rho} \xi_{T} k^{2} \cdot \frac{z \tau}{1+z \tau}\right] T_{k}(z) \\
& =\psi_{k}+\frac{\tau k^{2}}{1+z \tau}\left[\frac{p_{k}}{m \rho_{0}}-\left(\frac{C_{0}^{2}}{\gamma \rho_{0}}+\frac{\Delta}{\rho_{0}}\right) \rho_{k}\right. \\
& \left.-\left(\frac{C_{0}^{2} \beta_{T}}{\gamma}+\frac{\rho_{0}}{m} A_{\xi \xi \xi_{\rho} \xi_{T}}\right) T_{k}\right]
\end{aligned}
$$

and

$$
\begin{aligned}
& -\left[\frac{z(\gamma-1)}{\rho_{0} \beta_{T}}+\frac{z^{2} \tau T_{0} A_{\xi \xi} \xi_{\rho} \xi_{T}}{C_{v}(1+z \tau)}\right] \rho_{k^{\prime}}(z) \\
& +\left[z-\frac{\left(C_{I} / C_{v}\right) z^{2} \tau}{1+z \tau}+a k^{2}\right] T_{k^{\prime}}(z) \\
& =-\frac{z \tau}{1+z \tau}\left[\frac{T_{0} S_{k^{\prime}}}{C_{v} z \tau}+\left(\frac{\gamma-1}{\rho_{0} \beta_{T}}+\frac{T_{0} A_{\xi \xi} \xi_{\rho} \xi_{T}}{C_{v}}\right) \rho_{k}\right. \\
& \left.-\left(1-C_{I} / C_{v}\right) T_{k^{*}}\right] .
\end{aligned}
$$

Here we have made use of thermodynamic relationships which are developed in the appendix. The transformed quantities are

$$
\rho_{k}(z)=\int_{0}^{\infty} d t e^{-z t} \int_{v} d \mathbf{r} e^{i \mathbf{k} \cdot \mathbf{r}}\left[\rho(\mathbf{r}, t)-\rho_{0}\right] .
$$


$T_{k}(z)$ and $\psi_{k}(z)$ are obtained in the same way. In eq (16) $b_{0}=\left(\frac{4}{3} \eta_{s}+\eta_{v}\right) / m \rho_{0}, \quad C_{0}$ is the low-frequency sound speed, $\gamma$ is the ratio of specific heats,

$$
\Delta=\rho_{0}^{2} A_{\xi \xi} \xi_{\rho}^{2} / m,
$$

$\beta_{T}$ is the isothermal coefficient of thermal expansion, $\xi_{\rho}=-A_{\xi \rho} / A_{\xi \xi}$ and $\xi_{T}=-A_{\xi T} / A_{\xi \xi}$. In eq (17) $C_{I}=T_{0} A_{\xi \xi} \xi_{T}^{2}$ and $a=\lambda / \rho_{0} C_{r}$. The quantity $C_{I}$ represents the contribution of the internal degree of freedom to the specific heat when the density is held constant.

The right-hand sides of eqs (16) and (17) may be simplified by noting that in equilibrium

$$
\frac{1}{m \rho_{0}} d p=\frac{C_{0}^{2}}{\gamma \rho_{0}} d \rho+\frac{C_{0}^{2} \beta_{T}}{\gamma} d T
$$

and

$$
\frac{T_{0} d S}{C_{v}}=-\frac{\gamma-1}{\beta_{T} \rho_{0}} d \rho+d T .
$$

This simplification is permissible because we are interested in computing the correlation function $\left\langle\rho_{k} \rho_{-k_{i}}(z)\right\rangle$ in terms of the equilibrium correlation function $\left\langle\left|\rho_{k}\right|^{2}\right\rangle$.

To obtain $\left\langle\rho_{k} \rho_{-k}(z)\right\rangle$ we solve eqs $(15-17)$ for $\rho_{k}(z)$ in terms of the initial values $\rho_{k}, T_{k}$ and $\psi_{k}$. Only the terms involving $\rho_{k}$ are of interest because

$$
\left\langle\rho_{k_{i}} T_{-k_{i}}\right\rangle=\left\langle\rho_{k_{i}} \psi_{-k_{i}}\right\rangle=0 .
$$

\section{Comparison With a Calculation Using a Frequency Dependent, Single Relax- ation Time, Volume Viscosity}

\subsection{Thermal Relaxation}

We are interested in the case where the relaxing parameter is independent of the density, i.e., $\xi_{\rho}=0$. The correlation function is found to be

$$
\frac{\left\langle\rho_{k} \rho_{-k}(z)\right\rangle}{\left\langle\left|\rho_{k}\right|^{2}\right\rangle}=\frac{F(z)}{G(z)}
$$

where

$$
\begin{aligned}
F(z) & =z^{3} \tau\left(1-\frac{C_{I}}{C_{v}}\right) \\
& +z^{2}\left[1+a k^{2} \tau+b_{0} k^{2} \tau\left(1-C_{l} / C_{v}\right)\right] \\
& +z\left[a k^{2}+b_{0} k^{2}+a b_{0} k^{4} \tau+C_{0}^{2} k^{2} \tau\left(1-\frac{1}{\gamma}\right)\right] \\
& +a b_{0} k^{4}+C_{0}^{2} k^{2}(1-1 / \gamma)
\end{aligned}
$$

and

$$
\begin{aligned}
G(z) & =z^{4} \tau\left(1-C_{I} / C_{v}\right) \\
& +z^{3}\left[1+a k^{2} \tau+b_{0} k^{2} \tau\left(1-C_{I} / C_{v}\right)\right] \\
& +z^{2}\left[a k^{2}+b_{0} k^{2}+a b_{0} k^{4} \tau+C_{0}^{2} k^{2} \tau\left(1-C_{I} / C_{p}\right)\right] \\
& +z\left[a b_{0} k^{4}+C_{0}^{2} k^{2}\left(1+a k^{2} \tau / \gamma\right)\right] \\
& +a k^{2} C_{0}^{2} k^{2} / \gamma .
\end{aligned}
$$

For purposes of comparison let us recall the corresponding expressions derived earlier for the case of a frequency dependent volume viscosity [1]. There

$$
\begin{aligned}
\widetilde{F}(z) & =z^{3} \tilde{\tau}+z^{2}\left[1+\tilde{\tau}\left(a k^{2}+b_{0} k^{2}\right)\right] \\
& +z\left[a k^{2}+b_{0} k^{2}+b_{1} k^{2}+a b_{0} k^{4} \tilde{\tau}+C_{0}^{2} k^{2} \tilde{\tau}(1-1 / \gamma)\right] \\
& +a b_{0} k^{4}+a b_{1} k^{4}+C_{0}^{2} k^{2}(1-1 / \gamma) .
\end{aligned}
$$

and

$$
\begin{aligned}
\widetilde{G}(z) & =\tilde{\tau} z^{4}+z^{3}\left[1+\tilde{\tau}\left(a k^{2}+b_{0} k^{2}\right)\right] \\
& +z^{2}\left[a k^{2}+b_{0} k^{2}+b_{1} k^{2}+\tilde{\tau}\left(C_{0}^{2} k^{2}+a b_{0} k^{4}\right)\right] \\
& +z\left[C_{0}^{2} k^{2}+a b_{0} k^{4}+a b_{1} k^{4}+\tilde{\tau} C_{0}^{2} k^{2} a k^{2} / \gamma\right] \\
& +C_{0}^{2} k^{2} a k^{2} / \gamma .
\end{aligned}
$$

[Quantities with a tilde refer to the calculation in ref. 1.] Here $b_{0}$ is the nonrelaxing part of the kinematic viscosity, the relaxing part is $b_{1} /(1+i \omega \tilde{\tau})$

where

$$
b_{1}=\frac{\left(C_{p}-C_{v}\right) C_{I}}{\left(C_{v}-C_{I}\right) C_{p}} C_{0}^{2} \tau
$$

Direct comparison of $G$ and $\widetilde{G}$ shows that the two expressions are not equivalent. This may be realized by noting that $G$ contains the combination

$$
z^{3} b_{0} k^{2} \tau\left(1-C_{I} / C_{v}\right)+z^{2} C_{0}^{2} k^{2}\left(1-C_{I} / C_{p}\right) \tau
$$

while the corresponding terms in $\widetilde{G}$ are

$$
z^{3} b_{0} k^{2} \tilde{\tau}+z^{2} C_{0}^{2} k^{2} \tilde{\tau} .
$$

If we consider the limiting case where the internal degree of freedom provides the only loss mechanism for translational degrees of freedom it is possible to make $G$ and $\widetilde{G}$ into equivalent expressions. In this limit where $a=b_{0}=0$

$$
\begin{aligned}
G(z) & =z^{4} \tau\left(1-C_{I} / C_{v}\right)+z^{3} \\
& +z^{2} C_{0}^{2} k^{2} \tau\left(1-C_{I} / C_{p}\right)+z C_{0}^{2} k^{2}
\end{aligned}
$$

and 


$$
\begin{aligned}
\tilde{G}(z) & =z^{4} \tilde{\tau}+z^{3} \\
& +z^{2} C_{0}^{2} k^{2} \tau\left(1+\frac{C_{p}-C_{v}}{C_{v}-C_{l}} \cdot \frac{C_{I}}{C_{p}}\right)+z C_{0}^{2} k^{2} .
\end{aligned}
$$

A simple comparison shows that if

$$
\widetilde{\tau}=\tau\left(1-C_{l} / C_{v}\right)
$$

then $\widetilde{G}=G$ in this limit. It also can be shown that $\widetilde{F}=F$ under these conditions.

In an experiment what is measured is not $F(z) / G(z)$ but the real part of $F / G$ when $z=i \omega$ :

$$
\pi \frac{\left\langle\rho_{k \cdot \rho_{-k}}(\omega)\right\rangle}{\left\langle\left|\rho_{k}\right|^{2}\right\rangle}=\operatorname{Re} \frac{F(i \omega)}{G(i \omega)}
$$

A comparison of the complete expressions requires that we compare

$$
\operatorname{Re} \frac{F(i \omega)}{G(i \omega)} \text { with } \operatorname{Re} \frac{\tilde{F}(i \omega)}{\widetilde{G}(i \omega)}
$$

To do this we write

$$
\operatorname{Re} \frac{F(i \omega)}{G(i \omega)}=\operatorname{Re} \frac{N_{1}+i N_{2}}{D_{1}+i D_{2}}=\frac{N_{1} D_{1}+N_{2} D_{2}}{D_{1}^{2}+D_{2}^{2}}
$$

and

$$
\operatorname{Re} \frac{\widetilde{F}(i \omega)}{\widetilde{G}(i \omega)}=\frac{\widetilde{N}_{1} \widetilde{D}_{1}+\widetilde{N}_{2} \widetilde{D}_{2}}{\widetilde{D}_{1}^{2}+\widetilde{D}_{2}^{2}} .
$$

From eqs (22) and (23) it follows that

$$
\begin{aligned}
N_{1} & =-\omega^{2}\left[1+a k^{2} \tau+b_{0} k^{2} \tau\right]+a b_{0} k^{4}+C_{0}^{2} k^{2}(1-1 / \gamma) \\
N_{2} & =-\omega^{3} \tilde{\tau}+\omega\left[a k^{2}+b_{0} k^{2}+a b_{0} k^{4} \tau+C_{0}^{2} k^{2} \tau(1-1 / \gamma)\right] \\
D_{1} & =\omega^{4} \tilde{\tau}-\omega^{2}\left[a k^{2}+b_{0} k^{2}+a b_{0} k^{4} \tau+C_{0}^{2} k^{2} \tau\left(1-C_{1} / C_{p}\right)\right] \\
& +a k^{2} C_{0}^{2} k^{2} / \gamma
\end{aligned}
$$

and

$$
\begin{aligned}
D_{2} & =-\omega^{3}\left[1+a k^{2} \tau+b_{0} k^{2} \tau\right] \\
& +\omega\left[a b_{0} k^{4}+C_{0}^{2} k^{2}\left(1+\frac{a k^{2} \tau}{\gamma}\right)\right] .
\end{aligned}
$$

Here use has been made of eq (29).

The corresponding expressions for $\tilde{N}_{1}$, etc., are

$$
\begin{aligned}
\tilde{N}_{1} & =-\omega^{2}\left[1+a k^{2} \tau+b_{0} k^{2} \tau\right]+a b_{0} k^{4} \\
& +C_{0}^{2} k^{2}(1-1 / \gamma)+C_{0}^{2} k^{2} a k^{2} \tau \frac{C_{1}}{C_{v}}(1-1 / \gamma)
\end{aligned}
$$

$$
\begin{aligned}
\widetilde{N}_{2} & =-\omega^{3} \tilde{\tau}+\omega\left[a k^{2}+b_{0} k^{2}+a b_{0} k^{4} \tilde{\tau}+C_{0}^{2} k^{2} \tau(1-1 / \gamma)\right] \\
\widetilde{D}_{1} & =\omega^{4} \widetilde{\tau}-\omega^{2}\left[a k^{2}+b_{0} k^{2}+a b_{0} k^{4} \widetilde{\tau}+C_{0}^{2} k^{2} \tau\left(1-C_{l} / C_{p}\right)\right] \\
& +C_{0}^{2} k^{2} a k^{2} / \gamma
\end{aligned}
$$

and

$$
\begin{aligned}
\widetilde{D}_{2} & =-\omega^{3}\left[1+a k^{2} \tilde{\tau}+b_{0} k^{2} \tilde{\tau}\right] \\
& +\omega\left[C_{0}^{2} k^{2}\left(1+a k^{2} \frac{\tilde{\tau}}{\gamma}\right)+a b_{0} k^{4}\right. \\
& \left.+a k^{2} \tau C_{0}^{2} k^{2} \frac{C_{1}}{C_{r}}\left(1-\frac{1}{\gamma}\right)\right] .
\end{aligned}
$$

Examination of these expressions reveals; (a) they are not identical term for term, (b) there will be no significant difference in their values if

$$
\alpha=a k^{2} \tau C_{I} / C_{v}
$$

is small compared to 1 .

Using the parameters listed in ref. 1. we find that $\alpha$ is $6.4 \times 10^{-4}$ for $\mathrm{CCl}_{4}$ at $20{ }^{\circ} \mathrm{C}$ and is $2.0 \times 10^{-2}$ for $\mathrm{CS}_{2}$ at $20^{\circ} \mathrm{C}$. The wavevector was taken to be $10^{5} \mathrm{~cm}^{-1}$. This could be increased to $\alpha=0.2$ for $\mathrm{CS}_{2}$ if we consider scattering angles on the order of $180^{\circ}$ using the $\mathrm{He}-\mathrm{Ne}$ laser as the light source.

The spectra predicted by eqs (31) and (32) have been evaluated for $\mathrm{CCl}_{4}$ and $\mathrm{CS}_{2}$ using the parameters listed in ref. 1. For $\mathrm{CCl}_{4}$ there is no difference between the two curves. This is not surprising considering the small value of $\alpha$.

The two curves are not identical for

$$
\mathrm{CS}_{2}\left(k=3 \times 10^{5} \mathrm{~cm}^{-1}\right) .
$$

The central components are shown in figure 1. The

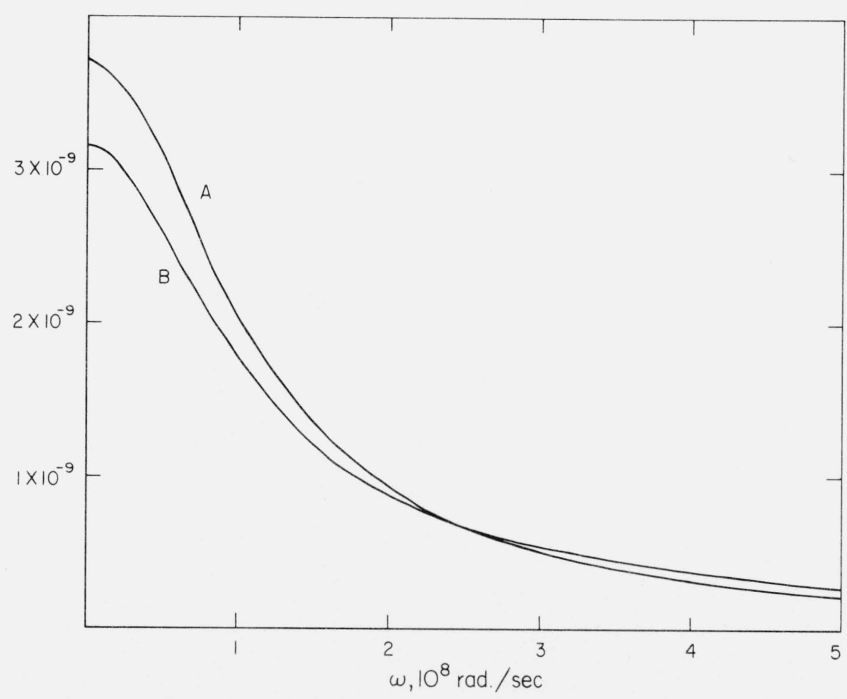

FIGURE 1. The central component of light scattered from C.S.2 as predicted by eq (32), curve $A$ and as predicted by eq (31) curve $B$. The parameters are taken from table of ref. 1 . The wave vector $l:=3 \times 10^{5} \mathrm{~cm}^{-1}$. 
curve labeled $A$ was obtained using a frequency dependent volume viscosity (eq 32 ). The curve labeled $B$ was obtained using the results of this calculation (eq 31). The difference in the amplitude at $\omega=0$ is due entirely to the term

$$
C_{0}^{2} k^{2} a k^{2} \tau \frac{C_{I}}{C_{v}}(1-1 / \gamma)
$$

in $\widetilde{N}_{1}$. Such a quantity is missing in $N_{1}$. It occurs as a result of multiplying the thermal diffusivity and the frequency dependent volume viscosity. We note for $\omega=0$, the present calculation yields the same amplitude as the theory which does not include internal relaxation processes.

The Brillouin components for $\mathrm{CS}_{2}$ are shown in figure 2. The differences are due to the relatively large value of $\alpha$ and to the different values of $\omega_{B} \tau$ where $\omega_{B}$ is the Brillouin frequency. For curve $A$, $\omega_{B} \tau=78$ while for curve $B, \omega_{B} \tau=113$. The large value of $\alpha$ is the dominant feature as $\omega_{B}$ depends most strongly on $k$ when $\omega_{B} \tau \gg 1$.

The possibility of finding a situation where $\alpha$ is appreciably greater than 0.2 appears to be remote. Longer relaxation times exist for rotational isomers [3], however, $C_{I} / C_{v}$ is very small so $\alpha$ is still much less than unity.

\subsection{Structural Relaxation}

Next let us examine briefly the other limiting case which involves a structural relaxation rather than a thermal relaxation. A purely structural relaxation occurs when $\xi_{T}=0$. The examination is speedily concluded by observing that if we formally identify $\Delta$ in eq (16) with $b_{1} / \tau=C_{x}^{2}-C_{0}^{2}$ (see eq (22) of ref. 1) then this case and the frequency dependent volume viscosity calculation yield identical results. Also no scaling of the relaxation times is needed.

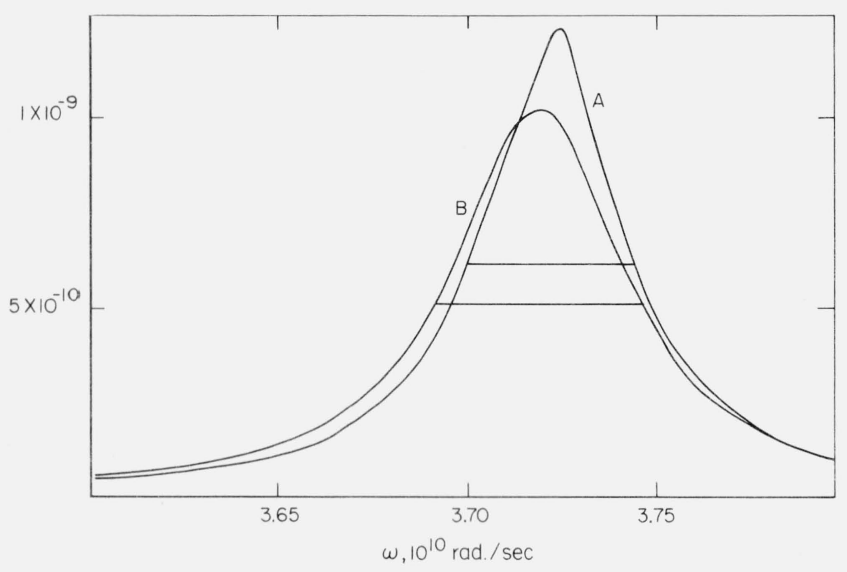

FIGURE 2. The Brillouin components of light scattered from $\mathrm{CS}_{2}$ as predicted by eq (32), curve $A$ and as predicted by eq (.31) curve $B$.

The parameters taken from table of ref. 1 . The wave vector $h=3 \times 10^{-5} \mathrm{~cm}^{-1}$. The horizontal lines indicate the full width at half maximum for the components.

\section{Summary and Discussion}

The frequency spectrum of density fluctuations in a fluid containing an internal degree of freedom has been calculated. The procedure used was to obtain an equation of motion for the internal variable $\xi$ by the methods of irreversible thermodynamics and then to solve the resulting set of coupled equations for the spectrum of the density fluctuations.

This calculation was compared with an earlier calculation which treated the internal degree of freedom as a frequency dependent volume viscosity rather than as a separate variable with an equation of motion of its own. Two limiting cases were examined. The structural relaxation case, $\xi_{T}=0$ was found to yield identical results when compared with the earlier calculation. The thermal relaxation case, $\xi_{\rho}=0$ does not agree exactly with the earlier calculation. The difference results from the ways the stresses due to the internal variable are treated. In the present calculation the stresses are additive only to first order [7]. In the earlier calculation they were strictly additive.

The equation of motion approach worked out in this paper has an intuitive appeal in that one can see directly how the internal variable enters the problem and how it is related to the temperature and the density. The introduction of a frequency dependent volume viscosity in the case of thermal relaxation is required by statistical mechanical considerations $[4,5]$ and is therefore the preferred way to handle internal degrees of freedom which are weakly coupled to the translational degrees of freedom.

This calculation could be adapted to a chemically reacting system. This aspect of fluctuation theory and light scattering is discussed at length in recent publications $[8,9]$ so we shall not discuss it here.

\section{Appendix}

Starting with eq (1) the differential expression for the Helmholtz free energy is

$$
d A=-S d T+\frac{p}{\rho_{0}^{2}} d \rho+A_{\xi} d \xi .
$$

The Maxwell relations implied by eq (36) are

$$
\begin{aligned}
& \left(\frac{\partial S}{\partial \xi}\right)_{T, \rho}=-A_{\xi T} \\
& \left(\frac{\partial S}{\partial \rho}\right)_{T, \xi}=-\frac{1}{\rho_{0}^{2}}\left(\frac{\partial p}{\partial T}\right)_{\rho, \xi}
\end{aligned}
$$

and

$$
\frac{1}{\rho_{0}^{2}}\left(\frac{\partial p}{\partial \xi}\right)_{\rho, T}=A_{\xi \rho}
$$


In equilibrium $A_{\xi}=0$ so that

$$
d A_{\xi}=A_{\xi \rho} d \rho+A_{\xi T} d T+A_{\xi \xi} d \xi=0 .
$$

It follows that

$$
A_{\xi \rho}=-A_{\xi \xi \xi} \xi_{\rho}
$$

and

$$
A_{\xi T}=-A_{\xi \xi \xi} \xi_{T}
$$

where $\xi_{\rho}=(\partial \xi / \partial \rho)_{T}$ and $\xi_{T}=(\partial \xi / \partial T)_{\rho}$.

If we combine these results with eqs (11) and (12) we obtain

$$
\begin{gathered}
\left(\frac{\partial p}{\partial \rho}\right)_{T, \xi}=\left(\frac{\partial p}{\partial \rho}\right)_{T}+\rho_{0}^{2} A_{\xi \xi} \xi_{\rho}^{2} \\
\left(\frac{\partial p}{\partial T}\right)_{\rho, \xi}=\left(\frac{\partial p}{\partial T}\right)_{\rho}+\rho_{0}^{2} A_{\xi \xi} \xi_{\rho} \xi_{T} \\
\left(\frac{\partial S}{\partial \rho}\right)_{T, \xi}=\left(\frac{\partial S}{\partial \rho}\right)_{T}-A_{\xi \xi \xi} \xi_{T} \xi_{\rho}
\end{gathered}
$$

and

$$
\left(\frac{\partial S}{\partial T}\right)_{\rho, \xi}=\left(\frac{\partial S}{\partial T}\right)_{\rho}-A_{\xi \xi} \xi_{T}^{2} .
$$

Finally we note that

$$
\frac{m C_{0}^{2}}{\gamma}=\left(\frac{\partial p}{\partial \rho}\right)_{T}
$$

$$
\frac{m \rho_{0} \beta_{T} C_{0}^{2}}{\gamma}=\left(\frac{\partial p}{\partial T}\right)_{\rho}
$$

and

$$
\left(\frac{\partial S}{\partial \rho}\right)_{T}=-\frac{C_{p}-C_{v}}{\rho_{0} T \beta_{T}} .
$$

A most interesting discussion with G. I. A. Stegeman provided the stimulus for this work.

\section{References}

[1] R. D. Mountain, J. Res. NBS 70A (Phys. and Chem.), No. 3, 207 (1966). Section 2 of this paper contains a summary of the formalism relating light scattering and density fluctuations.

[2] Karl F. Herzfeld and T. A. Litovitz, Absorption and Dispersion of Ultrasonic Waves (Academic Press, New York, 1959).

[3] S. R. de Groot and P. Mazur, Non-Equilibrium Thermodynamics (North Holland Publishing Co., Amsterdam, 1962).

[4] L. I. Komarov, Soviet Physics JETP 21, 99 (1965).

[5] Robert Zwanzig, J. Chem. Phys. 43, 714 (1965).

[6] V. T. Shmatov, Soviet Physics JETP 6, 1044 (1958).

[7] R. M. Mazo, J. Chem. Phys. 28, 1223 (1958).

[8] B. J. Berne and H. L. Frisch, J. Chem. Phys. 47, 3675 (1967).

[9] L. Blum and Z. W. Salsburg, J. Chem. Phys., to be published.

(Paper 72A1-486) 